Apidologie, 1971, 2 (4), 309-317.

\title{
EINFLUSS UNIPOLAR GELADENER LUFTIONEN AUF DIE MOTORISCHE AKTIVITÄT DER HONIGBIENEN
}

\author{
Influence des ions atmosphériques chargés de façon unipolaire \\ sur l'activité motrice des abeilles
}

Geza ALTMANN und Ulrich WARNKE

Zoologisches Institut des Universität des Saarlandes

Saarbrücken

\section{SUMMARY}

THE INFLUENCE OF UNIPOLAR AIR IONS ON THE ACTIVITY OF MOVEMENT OF THE HONEYBEE

The activity of the movement of honeybees was recorded according to the buzz by the wings in positive and negative ionized air, as well as under Faraday-condition.

1) The bees were of a lower aktivity under Faraday-condition. Frequently the bees were observed to fan for ventilation in collective manner.

2) The reaction upon exposure to negative and positive atmospheric ions followed immediatley. It was indicated by an increase of the amplitude during the move of the wings and by an increase of the activity of running.

3) The activity was higher in negative ionized air than in positive ionized air.

4) The turnover of water corresponded to the activity of movement under different conditions.

\section{ZUSAMMENFASSUNG}

Die motorische Aktivität der Bienen wird nach ihrer Lautäußerung in positiv und negativ ionisierter Luft, sowie unter völlig abgeschirmten Bedingungen (Faraday-Käfig) registriert. 1. Im Faraday-Käfig zeigen die Bienen eine geringe Aktivität. Es wird jedoch häufig ein gemeinsames Fächeln beobachtet. 
2. Die Reaktion auf negative und positive Luftionen tritt sofort auf und besteht in einer Vergrößerung der Flügelschlagamplitude und der Laufaktivität.

3. Die Aktivität in negativ geladener Luft ist größer als in positiv geladener Luft.

4. Der Wasserumsatz der Bienen entspricht, in gleicher Weise wie der Stoffwechsel, der jeweiligen motorischen Aktivität unter den verschiedenen Bedingungen.

\section{I. - EINLEITUNG}

Schon lange weiß man, daß die Honighiene besonders wetterempfindlich reagiert. Mehrere Untersuchungen (z. B. SCHUA, 1952, LeсомтE, 1954) zeigen eine direkte Korrelation des Verhaltens der Bienen zu bestimmten Wetterphasen. Am bekanntesten ist wohl das plötzliche Heimkehren der Sammelbienen und ihre Stechlust vor einem Gewitter. Welche Elemente einer Wetterkonstellation für das jeweilige Verhalten der Bienen verantwortlich sind, konnte bisher nicht befriedigend geklärt werden. Ein bisher kaum beachtetes meteorologisches Element ist das statische elektrische Feld, das durch die negative Oberflächenladung der Erde und die Summe der positiven Raumladungen der Ionosphäre aufgebaut wird. Altmann (1959) konnte feststellen, daß Bienen innerhalb eines künstlichen statischen Feldes von 400 Volt / Meter ihren Stoffwechsel erhöhen. Träger der Raumladungen sind Schwebeteilchen und die Gasmoleküle der Luft. Die Beweglichkeit und Größe dieser Ionen sind unterschiedlich. Im allgemeinen mißt man in Erdnähe ungefähr 750 positive und 600 negative mehrmolekulare Kleinionen pro Kubikmeter Luft. Die Zahlen variieren jedoch bei verschiedenen Wetterlagen und liegen vielfach erheblich höher. Der Einfluß dieser Ionen auf die motorische Aktivität der Bienen sollte im Labor untersucht werden, zumal zahlreiche diesbezügliche Untersuchungen an Wirbeltieren intensive $W$ irkungen bewiesen haben.

\section{II. - MATERIAL UND METHODE}

Alle untersuchten Bienen stammten aus dem Bienenstand des Zoologischen Institutes. der Universität Saarbrücken. Es wurden nur Flugbienen der Carnica-Rasse verwendet.

Der Versuchsaufbau wurde so gestaltet, daß alle Umweltbedingungen, d.h. meteorologischen Elemente, weitgehend konstant gehalten werden konnten (s. Abb. 1). Die gesamte Versuchseinrichtung befand sich in einem ca. $12 \mathrm{~m}^{3}$ großen Faraday-Raum aus einem geerdeten, doppelt gespannten Drahtgewebe. Dadurch konnten die Einflüsse des elektrischen Feldes der Atmosphäre weitgehend abgesehrimt werden. In Thermostaten (Th), bei einer Versuchstemperatur von $30^{\circ} \mathrm{C}$, wurden je 25 Bienen in durchlöcherten Käfigen aus Plexiglas von der Größe $7 \times 9 \times 4 \mathrm{~cm}$ gehalten. Nahrung und Wasser wurden in beliebiger Menge angeboten. Die relative Luftfeuchtigkeit in den Thermostaten betrug ca. $50 \%$. Alle Versuche wurden bei völliger Dunkelheit während der ganzen Versuchsdauer durchgeführt. Durch die mit den Thermostaten verbundenen Ionisatoren ( $\mathrm{Io}$ ) wurde die Luft in den Käfigen (K) bewegt und ionisiert. Zur Konstanterhaltung der Luftbewegung liefen die Ventilatoren in den Geräten während der ganzen Versuchsdauer. Der Ionen erzeugende Teil der Apparate wurde während der Versuche an- bezw. abgeschaltet. Der Geräuschpegel beider Ventilatoren wurde angeglichen. Während der Versuche herrschte völlige Ruhe. Die Lautäußerung der Bienen erfolgt hauptsächlich durch den Flügelschlag. Die Frequenz bestimmt die Haupttonhöhe, die Amplitude die Lautstärke. Die von den Bienen eines Versuchskäfiges erzeugten Töne der 


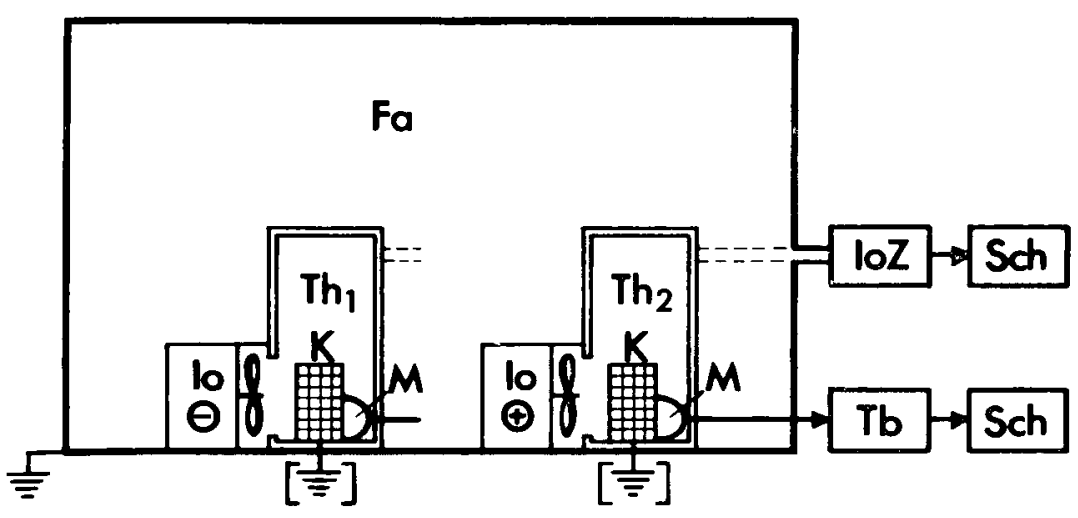

Авв. 1. - Schema des Versuchsaufbaus für die Messung der motorischen Aktivität der Honigbienen in ionisierter Luft.

$\mathbf{F a}=$ Faraday-Raum, Io = Ionisatoren, Ioz = Ionenzahlmeßgerät (Ionometer), $\mathrm{K}=$ Versuchskäfig, M. $=$ Mikrophon, Sch $=$ Schreiber, $\mathbf{T b}=$ Tonbandgerät, Th $=$ Thermostat.

FIg. 1. - Schêma du dispositif expérimental pour la mesure de l'activitê motrice de l'abeille dans l'air ionisé Fa : espace de Faraday, Io : ionisateurs, Ioz : ionomètre, $\mathrm{K}$ : cagette expérimentale, M : microphone, Sch : scripteur, Tb : magnétophone, Th : thermostat.

Flügelschläge wurden über ein Mikrophon (M) registriert. Ein 2-Kanal Tonbandgerät (Tb) erlaubte die gleichzeitige Aufnahme der Töne in beiden Versuchsapparaturen. Die Aufnahmeempfindlichkeit beider Kanäle wurde mit Hilfe eines Frequenz-Generators und eines Oszillographen angeglichen. Die Auswertung der Tonbänder erfolgte auf einem Schreiber (Sch) über integrierende R-C-Glieder. Die gemessene Spannung änderte sich mit der Flügelschlagfrequenz, der Amplitude des Flügelschlages und der Anzahl schwirrender und fächelnder Flügel.

Welche dieser aufgezählten Möglichkeiten für eine gemessene Spannungsänderung in Frage kommt, konnte durch Beobachtung des Verhaltens der Bienen im Käfig bei gleichzeitiger Aufnahme der Laute ermittelt werden. Auf diese Weise war es möglich die aufgezeichneten Meßspannungen zu “ eichen », d.h. die gemessenen Werte mit dem Verhalten der Bienen zu koordinieren. Wenn eine Biene am Mikrophon vorbeiläuft, gibt es einen Einzelausschlag des Schreibers und somit einen Strich auf dem Registrierpapier. Die Anzahl der kleinen Ausschläge pro Zeiteinheit gibt somit die Laufaktivität der einzelnen Bienen an. Rasch hintereinander folgende kleine Ausschläge bedeuten somit eine große Laufaktivität. Ein gemeinsames Fächeln vieler Bienen ohne Ortsbewegung bewirkt einen großen Ausschlag des Schreibers. Wenige große Ausschläge und dazwischen kleine Ausschläge des Schreibers bedeuten eine Änderung der Flügelschlagamplitude, verbunden mit Laufaktivität. Das bedeutet also eine größere Lautstärke des Tones der einzelnen vorbeilaufenden Bienen. In gleicher Weise wirkt sich auch die Schlagfrequenz der Flügel aus.

Ein Ionenzählgerät registrierte die Zahl der in den Versuchsthermostaten vorhandenen positiven bezw. negativen Luftionen. Die gleichzeitige Aufzeichnung der gemessenen Werte erfolgte ebenfalls mit Hilfe eines Schreibers. Ca. $10^{5} / \mathrm{cm}^{3}$ positive bezw. negative Kleinionen, in $50 \mathrm{~cm}$ Abstand von der Ionenquelle gemessen, wurden innerhalb des Faraday-Raumes von den Ionisatoren produziert. Dieser ionisierte Luftstrom wurde, wie auf der Abbildung ersichtlich, in die Versuchsthermostaten eingeleitet. Da die Faraday-Wände geerdet sind, stoßen sie negative Ionen $a b$, während positive Ionen an den $W$ änden ihre Ladung verlieren. Nach einer bestimmten Zeit sammeln sich deshalb negative Ionen innerhalb des Faraday-Raumes an (s. Abb. 2). Dabei kann es durch Ionisation von weiteren Gasmolekülen zu einer lawinenartigen Ionenvermehrung kommen (IsraEL, 1957). Die erzeugten kurzlebigen Kleinionen lagern sich an weitere Gasmoleküle und schwebende Partikel an und bilden so langlebige Mittel-bezw. Großionen, die noch mehr als 12 Stunden nach Abschalten der Ionisatoren nachgewiesen werden konnten. Um diese Ionen nicht bei abgestellter Ionenproduktion in die Versuchskäfige gelangen zu lassen, wurden über die Käfige noch zusätzlich geerdete Faraday-Schutzkäfige gestülpt, die bei veränderten Versuchsbedingungen wieder entfernt wurden.

Eine weitere Möglichkeit den Raum ionenfrei zu bekommen bestand darin, mit dem positiven Ionisator die Luft umzuwälzen, wobei durch die positiven Ionen die freien negativen Ionen rekombiniert wurden. 


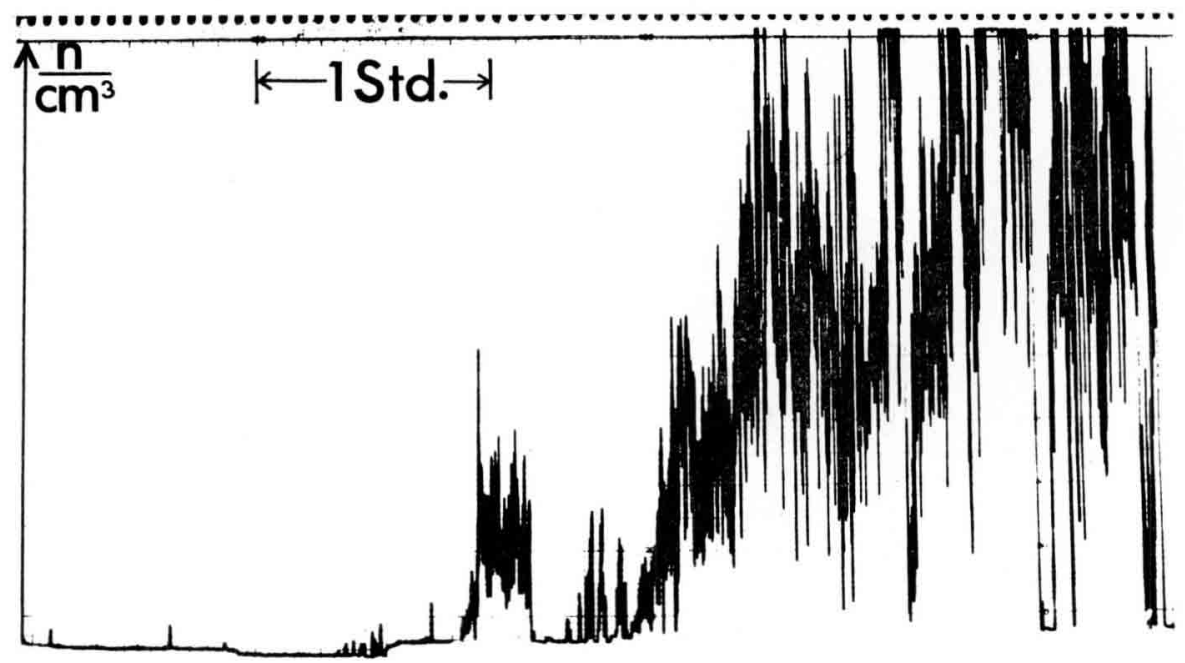

Aвв. 2. - Ansammlung von negativen Ionen innerhalb des Faraday-Raumes nach mehreren Stunden Versuchsdauer.

Fig. 2. - Accumulation d'ions négatifs à l'intérieur de l'espace de Faraday après plusieurs heures d'expérience.

Die Bienen wurden 24 Stunden vor Versuchsbeginn in ihre Käfige eingesetzt, damit sie sich an die neue Umgebung gewöhnen konnten. Beobachtungen bei Licht zeigten, daß sich die Tiere schon nach kurzer Zeit beruhigten und Futter nahmen. Die beschriebene Versuchsapparatur erlaubte auch die Registrierung der Aktivitätsphasen innerhalb von 24 Stunden. Circadiane Rhythmen der Aktivität zeigten sich auch bei den gekäfigten Bienen. Aus diesem Grunde war es notwendig sämtliche Versuche und Kontrollen gleichzeitig durchzuführen. Da immer zwei Gruppen von Bienen unter gleichen oder verschiedenen Versuchsbedingungen untersucht wurden, war die Sicherung der Ergebnisse gegeben.

\section{III. - ERGEBNISSE}

Obwohl Bienen im Käfig nicht fliegen, sind die Flügel doch fast immer in Bewegung. Sterzeln wurde bei den gekäfigten Bienen selten beobachtet, das Fächeln ist jedoch sehr häufig und dient allgemein zur Luftbewegung, insbesondere aber auch zur Lufterneuerung bei zu hohem Kohlendioxydgehalt. Man kann von dem Flügelschwirren ohne Lokomotion ein Flügelbewegen beim Umherlaufen unterscheiden. Diese Flügelbewegungen machten einen großen Teil der Tonaufzeichnungen aus, deren Grundton der Flügelschlagfrequenz entspricht (Fächeln : 120-150 Hz, Sterzeln 167-206 Hz bei einer Amplitude von 90-120. Wonlgemuth, 1960). Verschiedene Obertöne entstehen möglicherweise durch Eigenschwingungen der bewegten Flügel.

In völlig abgeschirmten Käfigen (Faraday-Bedingungen) kommt es zu einem einheitlichen Fächeln aller Versuchstiere, verbunden mit einer geringen Laufaktivität, was sich durch überwiegend langgezogene Ausschläge über die ganze Papierbreite des Schreibers ausdrückt. Die unregelmäßigen kleinen Ausschläge des Schreibers rühren von der geringen Laufaktivität der Bienen 
her (s. Abb. 5). Abbildungen 3 und 4 zeigen Ausschnitte aus den Originalprotokollen mit typischen Übergängen von Faraday-Bedingungen zur Einwirkung von positiv bezw. negativ ionisierter Luft. Die schematische Darstellung der Wirkung verschiedener Versuchsbedingungen zeigt Abbildung 5. Beim Wechsel von ionenfreier Luft im Faraday-Käfig zu positiv bezw. negativ

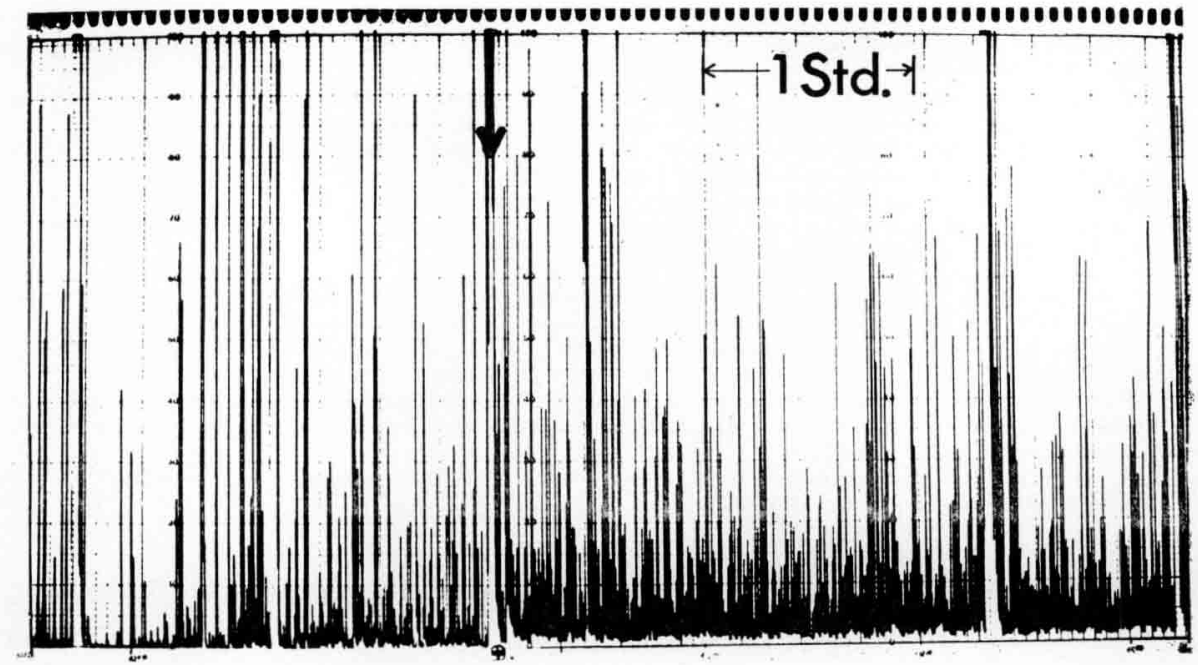

Aвв. 3. - Aufzeichnung der motorischen Aktivität der Versuchsbienen : Ubergang von Faraday - Bedingung $z u$ positiv ionisierter Luft.

Fıg. 3. - Graphique de l'activité motrice des abeilles expérimentales passage des conditions de Faraday à l'air ionisé positivement.

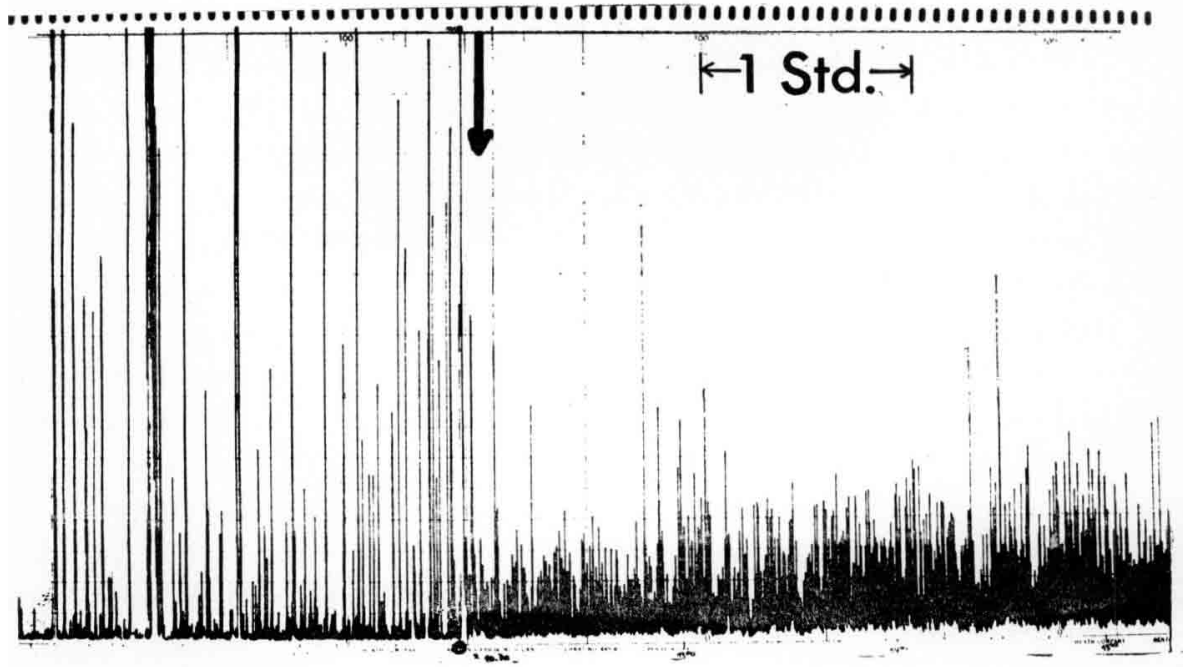

Aвв. 4. - Aufzeichnung der motorischen Aktivität der Versuchsbienen : Ubergang von Faraday - Bedingung zu negativ ionisierter Luft.

Fıg. 4. - Graphique de l'activité motrice des abeilles expérimentales : passage des conditions de Faraday à l'air ionisé négativement. 


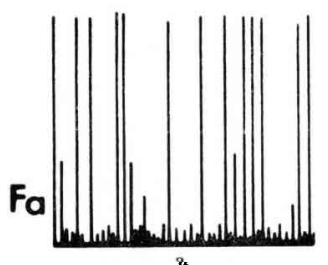

$(+1)$

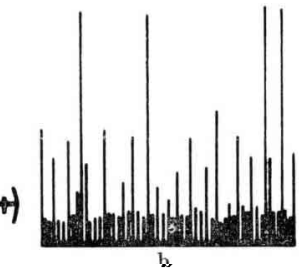

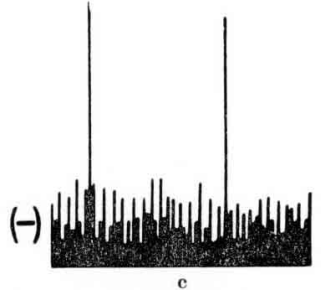

Aв8. 5. - Schematische Darstellung der Aktivitätsunterschiede der Versuchstiere unter Einwirkung von ionisierter Luft bzw. ohne Ionisation im Faraday - Raum.

a) Große Aussehläge des Schreibers : Einheitliches Fächeln aller Versuchstiere, verbunden mit einer geringen Laufaktivität : Häufige Situation in abgeschirmten Käfigen.

b) Wenige große Aussehläge und vermehrt kleine Ausschläge des Schreibers : Änderung der Flügelschlagamplitude, verbunden mit geringer Laufaktivität.

Häufige Situation unter Einwirkung positiv geladener Luftionen.

c) Zahlreiche kleine Ausschläge des Schreibers : Starke Laufaktivität der Bienen : Häufige Situation unter Einwirkung negativ geladener Luftionen.

Fig. 5. - Représentation schêmatique des différences d'activitê des abeilles expêrimentales sous l'action soit de l'air ionisé, soit des conditions régnant dans l'espace de Faraday, c'est-à-dire sans ionisation.

a) Grande amplitude des mouvements du seripteur : toutes les abeilles ventilent; l'activité locomotrice est faible. Situation fréquente dans les cages protégées.

b) Peu de grands mouvements du scripteur et nombreux petits mouvements : modification de l'amplitude des battements d'ailes liée à une faible activité locomotrice. Situation fréquente sous l'action d'ions atmosphériques positifs.

c) Nombreux petits mouvements du scripteur : forte activité locomotrice des abeilles. Situation fréquente sous l'action d'ions atmosphériques négatifs.

ionisierter Luft erfolgt sofort die entsprechende Reaktion. Die Lautstärke erhöht sich, d.h. die Amplitude des Flügelschlages vergrößert sich, wie außerdem andere Versuche an einzelnen Bienen im Windkanal bewiesen haben. Dazu erhöht sich die Laufaktivität der einzelnen Biene und außerdem hört das von allen Bienen gemeinsame Fächeln auf oder es verringert sich wesentlich. Unter Einwirkung negativ geladener Luftionen ist die Aktivitätsänderung am stärksten.

Die Wasserdampfabgabe (Transpiration) ist eng mit der Flügelaktivität verbunden. Fesselt man die Flügel einer Biene, so ist der Wasserverlust geringer, als bei Tieren, die normal fächeln (Lensky, 1964). Durch den Kohlendioxydgehalt der Luft wird die Atmungsintensität reguliert, d.h. die Stigmen werden mehr oder weniger geöffnet. Die Wasserdampfabgabe wurde von hochempfindlichen elektrischen Meßgeräten ${ }^{1}$ während der einzelnen Versuche registriert. Sie war am höchsten während der Einwirkung von negativ ionisierter Luft, schwächer bei positiv ionisierter Luft, während die geringsten Werte in Faraday-Käfigen gemessen wurden. Die höhere Wasserabgabe erforderte natürlich auch eine größere Wasseraufnahme, die jeweils zusammen mit der Zuckeraufnahme in 48 Stunden gemessen wurde. Das Gewicht der Rektalblase jeder Biene (insgesamt 80 Bienen je Versuch) gab Aufschluß über den Verbleib des aufgenommenen Wassers. Geht viel Wasser durch

1. Verwendet wurden Feuchtefühler der Fa. Weiss, Giessen. Die Fühler sind mit einer Spezial-LiClLösung getränkt. Gemessen wird unmittelbar die Temperatur des Taupunktes. Die Messung der Temperaturwerte im LiCl-Meßelement ist ein eindeutiges Maß der absoluten Lufteuchte, z. B. als Wasserdampfgehalt $\mathrm{g} / \mathrm{m}^{3}$ feuchte Luft. 
Transpiration verloren, so wird es aus dem Enddarm resorbiert. Bekanntlicherweise spielen dabei die Rektalpapillen eine sehr wesentliche Rolle. Vergleicht man die aufgenommene $W$ assermenge der Bienen mit dem Durchschnittsgewicht ihrer Rektalblasen, so erkennt man, daß in negativer Ionenluft eine vergleichsweise hohe Rückresorption stattgefunden hat. Die erhöhte Transpiration in negativ ionisierter Luft ist wahrscheinlich auf die höhere motorische Aktivität zurückzuführen und entspricht den bereits beschriebenen Ergebnissen (s. Abb. 6).

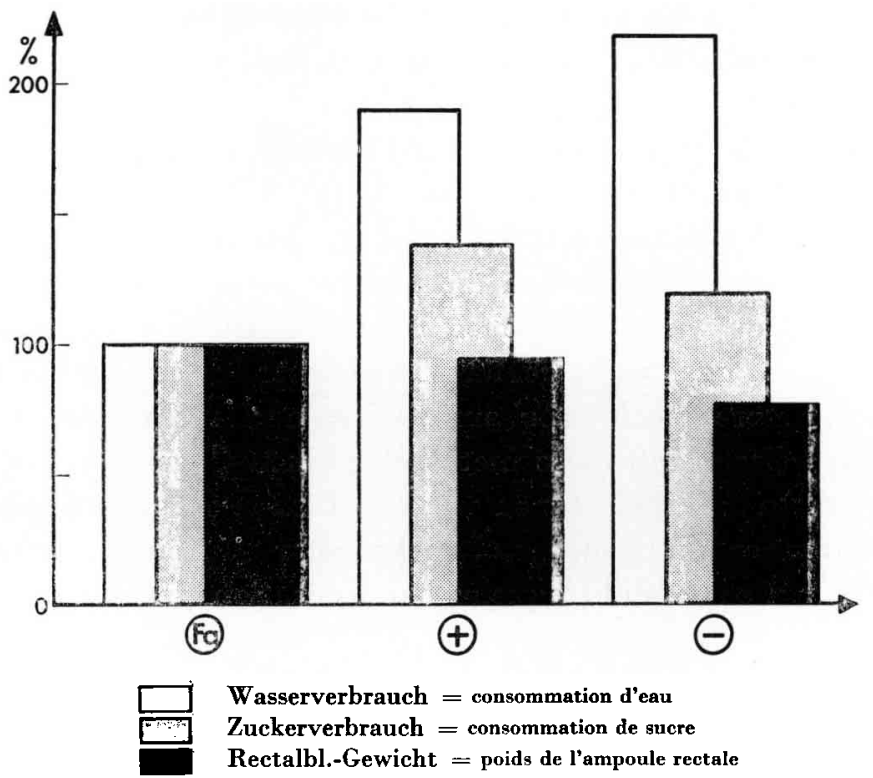

ABB. 6. - Vergleich der Nahrungs - und Wasseraufnahme und des Gewichtes der Rektalblasen unter verschiedenen Versuchsbedingungen (abgeschirmt, positiv ionisierte Luft, negativ ionisierte Luft). Durchschnittswerte von jeweils 80 Versuchstieren.

FIG. 6. - Comparaison des prises de nourriture et d'eau ainsi que du poids de l'ampoule rectale dans différentes conditions expérimentales (protégé, air ionisé positivement, air ionisé négativement). Valeurs moyennes pour, à chaque fois, 80 abeilles expérimentales.

\section{IV. - DISKUSSION}

Beobachtet man das Verhalten der Bienen im Freien und registriert die Anzahl der unipolaren atmosphärischen Ionen, so scheint sich eine Beziehung herauszustellen. Bei einer außergewöhnlich großen Anzahl negativer Ionen sind Bienen (z. B. Wächterinnen) besonders reizbar und angriffslustig gegenüber Bienen anderer Völker und auch dunkle oder schwarze Flächen werden sehr häufig angegriffen. Nach Untersuchungen von Norinder und SiksNA (1949) erhöht sich die Anzahl negativer Ionen vor einem Regen und während des Regens beträchtlich. Dieses Phänomen konnte durch unsere Messungen 
bestätigt werden. Ein außergewöhnlich großer Anstieg von negativen Ionen zeigt sich bei Gewittern. Die negativen Ionen stiegen von 800 auf 7000 pro $\mathbf{c m}^{3}$ vor einem Gewitterregen. Zur gleichen Zeit stieg auch die Zahl der positiven Ionen, jedoch weniger stark. Die Konzentration der Luftionen normalisierte sich nach dem Gewitter wieder sehr rasch. Möglicherweise ist somit die U̇berzahl negativer oder positiver Ionen während bestimmter Wetterphasen die Ursache für die starke Reizbarkeit der Bienen. Eingehendere Untersuchungen im Freien stehen noch aus. Es bleibt noch festzustellen, in welchem Ausmaß die Steigerung der Aktivität in negativ ionisierter Luft mit einer Erhöhung der Aggression verbunden ist. In diesem Zusammenhang wird auch die Abhängigkeit der Sammelleistung der Bienen von den genannten physikalischen Faktoren geprüft.

Arbeiten von Krueger und Sмith (1960) beweisen einen tiefgreifenden physiologischen Einfluß der ionisierten Luft. Normalerweise ist Sauerstoff negativ und Kohlendioxyd positiv geladen. Analog zu den Versuchen an Säugern scheinen auch bei Bienen die negativ aufgeladenen Sauerstoff-Moleküle stimulierend zu wirken. Welchen Effekt positiv geladene KohlendioxydMoleküle haben, ist noch nicht geklärt. In gleicher Weise ist die Art der Rezeption für diese Faktoren noch unbekannt. Erste, noch nicht gesicherte Versuche scheinen zu beweisen, daß diese meteorologischen Gegebenheiten der Bienen mit den Antennen wahrgenommen werden. Bienen reagierten nach Amputation ihrer Antennen im Windkanal nicht mehr mit einer Veränderung der Flügelschlag amplitude und-frequenz bei Ionisation der Luft.

Eingegangen im April 1971.

Reçu pour publication en avril 1971.

\section{RÉSUMÉ}

Un nombre déterminé d'abeilles butineuses (à chaque fois 25) appartenant à la race carniolienne ont été soumises à de l'air ionisé soit négativement soit positivement et leur comportement comparé à celui d'abeilles témoins maintenues dans des conditions de protection absolue (cage de Faraday). Toutes les expériences ont eu lieu dans un espace de Faraday de $12 \mathrm{~m}^{\mathrm{a}} \mathrm{de}$ façon à éliminer complètement toute in fluence électrique météorologique extérieure. On a étudié principalement l'activité motrice des abeilles dans les conditions précisées ci-dessus.

Les abeilles se trouvaient dans deux enceintes thermostatées à $30^{\circ} \mathrm{C}$ et à l'obscurité complète. Ces enceintes étaient reliées à des ionisateurs chargeant l'air soit positivement soit négativement et assurant sa circulation dans l'espace intérieur.

La quantité d'ions atmosphériques unipolaires actifs, positifs ou négatifs, dans les enceintes thermostatées était d'environ $10^{5} / \mathrm{cm}^{3}$.

Bien que les abeilles ne volent pas dans la cage, leurs ailes sont constamment en mouvement. Les sons émis par le battement des ailes des abeilles en cage étaient enregistrés par l'intermédiaire d'un microphone sur un magnétophone stéréophonique dont les canaux étaient équilibrés pour la sensibilité. A partir de la bande magnétique les sons émis par les abeilles étaient enregistrés graphiquement après transformation en tensions mesurables par deux éléments accordés. 
Il est possible de distinguer la ventilation d'avec le battement d'ailes qui aide les allées et venues. Pour tous les mouvements d'ailes il se produit un son fondamental qui correspond à la fréquence des battements. L'amplitude des battements conditionne la force du son.

Les figures 3, 4 et 5 laissent apparaître les phénomènes suivants. Chaque changement des facteurs physiques provoque une réaction immédiate. L'activité locomotrice de chaque abeille augmente dans l'air ionisé tandis que dans des conditions de protection la ventilation entreprise par toutes les abeilles cesse ou bien diminue fortement. L'action la plus forte apparait dans l'air ionisé négativement. Dans l'air ionisé positivement l'amplitude des battements d'ailes des abeilles est particulièrement grande tandis que leur activité locomotrice n'atteint pas sa valeur maximale.

La transpiration est étroitement liée à l'activité alaire. La perte d'eau est extrêmement faible chez des abeilles dont les ailes sont entravées. Au cours de nos expériences la transpiration a été enregistrée au moyen de capteurs électriques très sensibles. Les graphiques montrent une décroissance progressive des valeurs mesurées à partir des abeilles en atmosphère ionisée négativement jusqu'aux abeilles maintenues dans un espace de Faraday en passant par celles qui sont en atmosphère ionisée positivement.

L'augmentation de la transpiration nécessita une plus grande consommation d'eau qui fut enregistrée avec la consommation de sucre pour une unité de temps de 48 heures. Le poids de l'ampoule rectale de chaque abeille donna des indications sur le reliquat de l'eau absorbée. Si beaucoup d'eau est perdue par la transpiration elle est résorbée à partir de l'intestin postérieur. Si l'on compare la quantité d'eau absorbée par les abeilles avec le poids moyen de leur ampoule rectale on remarque qu'en atmosphère ionisée négativement une résorption relativement plus forte a eu lieu. L'augmentation de la transpiration dans l'air ionisé négativement est probablement à rapprocher de l'élévation de l'activité motrice et correspond bien aux résultats décrits ici.

\section{LITERATUR}

Altmanv G., 1957. Untersuchungen über den Wasserhaushalt der Honigbiene. Annales Universitatis Saraviensis, III, 1 .

Altmann G., 1959. Der Ein fluß statischer elektrischer Felder auf den Stoffwechsel von Insekten. Z.f. Bienenforsch., 4, 10.

ISRAEL H.. 1957. Luftelektrizität und Radioaktivität. Springer-Verlag.

Krueger A. P. u. R. F. Smith, 1960. The biological Mechanisms of Air Ion Action. J. of Gen. Physiol., 43, 3, 533-540. J. of Gen. Physiol., 44, 2, 269-276.

Leсомте J., 1954. Essai d'une Analyse causale du Comportement agressif des ouvrières d'abeilles (Apis mellifica). Insectes sociaux, 1, 49-57.

Lensky Y., 1964. L'économie de liquides chez les abeilles aux températures élevées. Insectes sociaux, 11, 207-222.

Norinder H. u. R. Siksna, 1948. Variation characteristics of the density of small-ions. Arkiv for Geofysik, 1, 1, 1-31.

SCHUA L., 1952. Untersuchungen über den Ein fluß meteorolog. Elemente auf das Verhalten der Honigbienen. Z. vergl. Physiol., 34, 258-277.

Wонцgemutн R., 1960. Ü̉ber das Fächeln der Bienen und dessen Verhältnis zum Fliegen. Z. vergl. Physiol., 43, 615-64.1.

Wohlgemutr R., 1962. Die Schlagform des Bienen flügels beim Sterzeln im Vergleich zur Bewegungsweise beim Fliegen und Fächeln. Z. vergl. Physiol., 45, 581-589. 